\title{
Appendix 7: Template of Therapist Adherence Checklist
}

\author{
Transactional Analysis Psychotherapy for Depression - \\ Therapist Adherence Checklist
}

\section{Therapist Adherence Scale.}

Therapist:

Client Code:

Session Number:

Date:

The twelve therapeutic tasks listed below constitute the essential core treatment plan for depression. Please tick next to each item to indicate whether you attended to this task in the therapy session and give yourself a score using the six-point rating scale below for each item. If the item is not applicable, please circle the N/A option. In the notes section, under each item and before the scale, please indicate how far you and the client achieved that item. Please also indicate with an asterisk which three items you focused on most in the session

1. Much improvement in application needed: I felt like a beginner, as if I didn't have the concept.

2. Moderate improvement needed: I seemed like an advanced beginner, who is beginning to do this, but needs to work on the concept more.

3. Slight improvement in application needed: I need to make a focused effort to do more of this.

4. Adequate application of principle: I did enough of this, but need to keep working on improving how well I do it.

5. Good application of principle: I did enough of this and did it skillfully.

6. Excellent application of principle: I did this consistently and even applied it in a creative way.

\section{Key Therapeutic Tasks in Transactional Analysis Treatment of Depression}

1. Create an 'I'm OK- You're OK' relationship where the client feels safe enough to explore their thoughts, feelings and experiences and begin to internalise the experience of being accepted

Notes:

\begin{tabular}{|c|c|c|c|c|c|c|}
\hline $\begin{array}{l}\text { Much } \\
\text { improvement } \\
\text { needed }\end{array}$ & $\begin{array}{l}\text { Moderate } \\
\text { improvement } \\
\text { needed }\end{array}$ & $\begin{array}{l}\text { Slight } \\
\text { improvement } \\
\text { needed }\end{array}$ & Adequate & Good & Excellent & Not Applicable \\
\hline 1 & 2 & 3 & 4 & 5 & 6 & $\mathrm{~N} / \mathrm{A}$ \\
\hline
\end{tabular}


2. Identify, reflect upon the origins of and re-evaluate self-critical ego state dialogue

Notes:

\begin{tabular}{|c|c|c|c|c|c|c|}
\hline $\begin{array}{l}\text { Much } \\
\text { improvement } \\
\text { needed }\end{array}$ & $\begin{array}{l}\text { Moderate } \\
\text { improvement } \\
\text { needed }\end{array}$ & $\begin{array}{l}\text { Slight } \\
\text { improvement } \\
\text { needed }\end{array}$ & Adequate & Good & Excellent & Not Applicable \\
\hline 1 & 2 & 3 & 4 & 5 & 6 & N/A \\
\hline
\end{tabular}

3. Identify, re-evaluate and challenge contaminations and script beliefs which negatively impact on the individual's self-concept and expectations of others and life

Notes:

\begin{tabular}{|c|c|c|c|c|c|c|}
\hline $\begin{array}{l}\text { Much } \\
\text { improvement } \\
\text { needed }\end{array}$ & $\begin{array}{l}\text { Moderate } \\
\text { improvement } \\
\text { needed }\end{array}$ & $\begin{array}{l}\text { Slight } \\
\text { improvement } \\
\text { needed }\end{array}$ & Adequate & Good & Excellent & Not Applicable \\
\hline 1 & 2 & 3 & 4 & 5 & 6 & $\mathrm{~N} / \mathrm{A}$ \\
\hline
\end{tabular}

4. Support the individual to recognise, re-evaluate and challenge self-limiting systems of thinking, behaviour and experience which maintain the depression (racket system)

Notes:

\begin{tabular}{|c|c|c|c|c|c|c|}
\hline $\begin{array}{l}\text { Much } \\
\text { improvement } \\
\text { needed }\end{array}$ & $\begin{array}{l}\text { Moderate } \\
\text { improvement } \\
\text { needed }\end{array}$ & $\begin{array}{l}\text { Slight } \\
\text { improvement } \\
\text { needed }\end{array}$ & Adequate & Good & Excellent & Not Applicable \\
\hline 1 & 2 & 3 & 4 & 5 & 6 & $\mathrm{~N} / \mathrm{A}$ \\
\hline
\end{tabular}

5. Explore, reflect upon and change stroking patterns (accepting positive strokes, giving self positive strokes, reduction in negative self-stroking/ self-criticism)

Notes:

\begin{tabular}{|c|c|c|c|c|c|c|}
\hline $\begin{array}{l}\text { Much } \\
\text { improvement } \\
\text { needed }\end{array}$ & $\begin{array}{l}\text { Moderate } \\
\text { improvement } \\
\text { needed }\end{array}$ & $\begin{array}{l}\text { Slight } \\
\text { improvement } \\
\text { needed }\end{array}$ & Adequate & Good & Excellent & Not Applicable \\
\hline 1 & 2 & 3 & 4 & 5 & 6 & $\mathrm{~N} / \mathrm{A}$ \\
\hline
\end{tabular}

6. Identify and challenge discounting and grandiosity (e.g 'if things go wrong it is my fault' - discounts external factors and is grandiose about role of self)

Notes:

\begin{tabular}{|c|c|c|c|c|c|c|}
\hline $\begin{array}{l}\text { Much } \\
\text { improvement } \\
\text { needed }\end{array}$ & $\begin{array}{l}\text { Moderate } \\
\text { improvement } \\
\text { needed }\end{array}$ & $\begin{array}{l}\text { Slight } \\
\text { improvement } \\
\text { needed }\end{array}$ & Adequate & Good & Excellent & Not Applicable \\
\hline 1 & 2 & 3 & 4 & 5 & 6 & $N / A$ \\
\hline
\end{tabular}


7. Support the reflection upon and re-evaluation of life experiences that have contributed to a sense of worthlessness

Notes:

\begin{tabular}{|c|c|c|c|c|c|c|}
\hline $\begin{array}{l}\text { Much } \\
\text { improvement } \\
\text { needed }\end{array}$ & $\begin{array}{l}\text { Moderate } \\
\text { improvement } \\
\text { needed }\end{array}$ & $\begin{array}{l}\text { Slight } \\
\text { improvement } \\
\text { needed }\end{array}$ & Adequate & Good & Excellent & Not Applicable \\
\hline 1 & 2 & 3 & 4 & 5 & 6 & $\mathrm{~N} / \mathrm{A}$ \\
\hline
\end{tabular}

8. Support the individual to make new decisions about how they will view themselves, relate to others and engage with the world

Notes:

\begin{tabular}{|c|c|c|c|c|c|c|}
\hline $\begin{array}{l}\text { Much } \\
\text { improvement } \\
\text { needed }\end{array}$ & $\begin{array}{l}\text { Moderate } \\
\text { improvement } \\
\text { needed }\end{array}$ & $\begin{array}{l}\text { Slight } \\
\text { improvement } \\
\text { needed }\end{array}$ & Adequate & Good & Excellent & Not Applicable \\
\hline 1 & 2 & 3 & 4 & 5 & 6 & $\mathrm{~N} / \mathrm{A}$ \\
\hline
\end{tabular}

9. Support the deconfusion process whereby the individual identifies, expresses and reflects upon repressed feelings (including repressed anger and working through of grief and loss)

Notes:

\begin{tabular}{|c|c|c|c|c|c|c|}
\hline $\begin{array}{l}\text { Much } \\
\text { improvement } \\
\text { needed }\end{array}$ & $\begin{array}{l}\text { Moderate } \\
\text { improvement } \\
\text { needed }\end{array}$ & $\begin{array}{l}\text { Slight } \\
\text { improvement } \\
\text { needed }\end{array}$ & Adequate & Good & Excellent & Not Applicable \\
\hline 1 & 2 & 3 & 4 & 5 & 6 & $N / A$ \\
\hline
\end{tabular}

10. Support the individual to explore and experiment with new ways of relating to others which enhance self-worth

Notes:

\begin{tabular}{|c|c|c|c|c|c|c|}
\hline $\begin{array}{l}\text { Much } \\
\text { improvement } \\
\text { needed }\end{array}$ & $\begin{array}{l}\text { Moderate } \\
\text { improvement } \\
\text { needed }\end{array}$ & $\begin{array}{l}\text { Slight } \\
\text { improvement } \\
\text { needed }\end{array}$ & Adequate & Good & Excellent & Not Applicable \\
\hline 1 & 2 & 3 & 4 & 5 & 6 & $\mathrm{~N} / \mathrm{A}$ \\
\hline
\end{tabular}

11. Designing and negotiating behavioural contracts such as awareness exercises homework, self-care contracts, exercise, diet and sleep hygiene contracts.

Notes:

\begin{tabular}{|c|c|c|c|c|c|c|}
\hline $\begin{array}{l}\text { Much } \\
\text { improvement } \\
\text { needed }\end{array}$ & $\begin{array}{l}\text { Moderate } \\
\text { improvement } \\
\text { needed }\end{array}$ & $\begin{array}{l}\text { Slight } \\
\text { improvement } \\
\text { needed }\end{array}$ & Adequate & Good & Excellent & Not Applicable \\
\hline 1 & 2 & 3 & 4 & 5 & 6 & $N / A$ \\
\hline
\end{tabular}

12. Facilitate the client's attachment to and engagement with life, others and the world

Notes:

\begin{tabular}{|c|c|c|c|c|c|c|}
\hline $\begin{array}{l}\text { Much } \\
\text { improvement } \\
\text { needed }\end{array}$ & $\begin{array}{l}\text { Moderate } \\
\text { improvement } \\
\text { needed }\end{array}$ & $\begin{array}{l}\text { Slight } \\
\text { improvement } \\
\text { needed }\end{array}$ & Adequate & Good & Excellent & Not Applicable \\
\hline 1 & 2 & 3 & 4 & 5 & 6 & $N / A$ \\
\hline
\end{tabular}

